\title{
RIOD (Red Iberoaméricana de ONGs que trabajan en drogodependencias)
}

\author{
Catalá, T. \\ Presidente de la RIOD y presidente de la Asociación Proyecto \\ Enviar correspondencia a: \\ Bartomeu Catalá Barceló. C/ Oblates, 2307011 Palma de Mallorca. Tel. 971793750
}

\section{RESUMEN:}

La RIOD (Red iberoamericana de Ongs que trabajan en drogodependencias) se crea en mayo de 1999 con el apoyo del Plan Nacional sobre Drogas español y de la AECl (Agencia Española de Cooperación Internacional). Está formada por 59 asociaciones de 19 países latinoamericanos que trabajan en el campo de la prevención, tratamiento y reinserción de las drogodependencias. Funciona con una estructura democrática con una asamblea, una junta directiva, unos nodos regionales, una secretaría técnica y grupos de trabajo.

Desde su creación ha organizado tres seminarios iberoamericanos y tres seminarios regionales y ha participado en diversos foros. Tiene una web (www.riod.net o www.riod.org) y ha elaborado diversos documentos de trabajo. En estos momentos se trabaja en mejorar la profesionalización, la adecuación de las actividades a las distintas realidades y en la evaluación de los acciones.

Palabras clave: redes, tratamiento, prevención, ong, Latinoamérica, drogas.

\section{ABSTRACT}

The RIOD (South American Network of NGO's working with Drug Addicts)was created in May 1999 with the support of the Spanish National Drug Plan and the AECI (Spanish International Cooperation Agency). It comprises 59 associations in 19 South American countries working in the field of prevention, treatment and reinsertion of drug addicts. It has a democratic structure with an assembly, board of directors, regional nodes, secretariat and working groups.

Since its creation, it has organised three South American seminars and three regional seminars and has taken part in several forums. It has a website (www.riod.net or ww.riod.org) and has drawn up several working documents. At the present time, it is working to improve professionalism, adapting activities to suit reality and evaluation of actions undertaken.

Key words: networks, treatment, prevention, NGO, South America, Latin America, drugs, collaboration
$\mathbf{E}$ I Plan Nacional sobre Drogas de España y la AECl (Agencia Española de Cooperación Internacional) realizaron en abril de 1998, en Cartagena de Indias, Colombia, el "I Seminario Iberoamericano sobre Cooperación en materia de Drogodependencias", al cual fueron invitadas 50 ONGs de las áreas de investigación, formación, prevención y tratamiento en el área de las drogodependencias de España y 18 países latinoamericanos.

El Seminario facilitó el encuentro por primera vez de estas 50 ONGs. En la "Declaración de Cartagena", suscrita por la totalidad de los asistentes, las entidades anunciaron su decidido compromiso por impulsar la creación de una red lberoamericana que se convirtiera en espacio permanente de encuentro para optimizar cada una de las acciones que se realizan, clarificar la seriedad de las ONGs y potenciar su aportación a la sociedad.

No hacía mucho que se había creado RIPRES, potenciada por el Departamento de Estado de USA, a la cual pertenecen instituciones privadas y públicas, que trabajan en prevención. Creímos importante que la RIOD se centrara en ONGs y que abarcara la prevención y el tratamiento. Por ello uno de los primeros problemas que tuvimos fue el de clarificar quiénes podían pertenecer y quiénes no

Todos éramos conscientes del papel importante que en España y en Latinoamérica las ONGs realizan y han realizado en cuanto a prevención, tratamiento y rehabilitación de drogodependencias y la gran aportación que hacen desde la profesionalidad y desde el voluntariado. Sin duda la respuesta de la sociedad civil a través de las ONGs en este problema no sólo es de 
capital importancia sino totalmente necesaria, ya que donde no pueden llegar los gobiernos, están presentes ellas desde hace tiempo. Y esto además de constancia, supone, muchas veces, que se trabaja desde unas grandes limitaciones, pero también desde una gran cercanía a los problemas y con respuestas adecuadas. Al mismo tiempo sabemos de la posibilidad de que surjan ONGs con buena voluntad, pero con poca profesionalidad; o bien que surjan otras cuyo único interés sea el lucro, y en realidad sólo sean empresas de servicio.

Es en esta gama de posibilidades y de problemas, dentro de una realidad global como la estamos viviendo la respuesta de la sociedad civil no puede menos de ser globalizadora. Y esto en lenguaje de ONGs quiere decir trabajar no solos, sino en red. Una red que se crea porque se comparten unas mismas demandas y necesidades, problemas, lengua, cultura... y también porque se necesita apoyo en la gestión económica y organizativa. Esta red es no lucrativa y privada y queremos que el estilo de coordinación no sea ni vertical, ni carismática, ni asambleista, sino democrática. Y que nuestra red no sea ni una multinacional, ni una simple suma de siglas, sino desde la consciencia de que nos enfrentamos a problemas y necesidades comunes poder así enriquecernos mutuamente e ir realizando los objetivos que nos marcamos en nuestra Asamblea Constituyente en mayo de 1999 en el Escorial con un total de 65 ONGs de 19 paises.

Somos sabedores de la potencialidad de las ONGs, pero también de los peligros; por ello uno de los primeros puntos que hemos trabajado ha sido el de consensuar unos mínimos criterios de calidad tanto en prevención como en tratamiento, tanto a nivel programático como en el de gestión y queremos que estos dos documentos sirvan de base para el diseño y validación de los programas propuestos y realizados a nivel interno por las ONG miembros de la RIOD. Igualmente será imprescindible suscribir explícitamente y llevarlo a la práctica por parte de las ONG integrantes de la RIOD. Por otra parte estamos elaborando el Plan Estratégico que esperamos aprobarlo en la Asamblea de Agosto de este año y de esta forma tener unos objetivos y estrategias para estos próximos cuatro años.

\section{OBJETIVOS}

Estimular el fortalecimiento institucional, tanto de la RIOD como de cada uno de sus miembros.

Generar un espacio de encuentro y comunicación para compartir informaciones, experiencias y saberes.

Articular la interlocución entre los miembros de la RIOD y las instituciones financiadoras, favorecien- do la transferencia de información sobre fuentes y métodos de financiación.

Contribuir a la formación de profesionales.

Facilitar el intercambio de profesionales entre las entidades miembros de la RIOD

Potenciar la realización de estudios y la investigación en materia de drogodependencias.

Favorecer las acciones de cooperación para el desarrollo de la RIOD y sus miembros.

Interlocución con gobiernos y entidades multilaterales e internacionales.

\section{LA ESTRUCTURA}

Se han definido los miembros fundadores de RIOD: 59 ONG de 19 países. En el camino han quedado varias ONG que no cumplieron los requisitos mínimos: presentar estatutos, certificado de registro público y una carta de su junta directiva aceptando los estatutos y fines de RIOD. Se tienen unos Estatutos, aprobados por Asamblea General en 1999 y reformados para mejorarlos en Asamblea General en 2000. Se cuenta con 2 Miembros de Honor: Delegación del Gobierno para el Plan Nacional sobre Drogas de España y CICAD, Comisión Interamericana para el Control del Abuso de Drogas de la OEA. Se han acordado en Asamblea General los criterios de ingreso, para abrir la Red a muchas otras entidades, siempre y cuando cumplan unos requisitos mínimos que tienen que ver con aspectos éticos, científicos, técnicos y administrativos. Se ha invitado a todos los gobiernos de lberoamérica para que sean Miembros Observadores de RIOD.

Se cuenta con un Reglamento Interno, que define las reglas de juego operativas en la Red. También fue aprobado por Asamblea, después de un año de elaboración y consultas. Se funciona con un esquema sencillo:

- Asamblea General, donde cada entidad tiene un voto.

- Junta Directiva, elegida con dos mecanismos: 4 vocales, que son elegidos por cada uno de los 4 nodos geográficos, y 3 miembros elegidos por toda la Asamblea, para Presidencia, Tesorería y Secretaría.

- 4 Nodos: Centroamérica y Caribe, Andino, Cono Sur y España.

- Secretaría Técnica: con sede en la Presidencia de RIOD, actualmente en Madrid.

- Comisiones temporales para acciones puntuales.

Se cuenta con un presupuesto propio, producto de las cuotas anuales de membresía que pagan todos los miembros, equivalente al salario mensual mínimo legal en cada país. 


\section{LAS ACCIONES}

- Declaración de Cartagena de Indias: un documento donde se reclama de los gobiernos y organismos multilaterales un equilibrio entre las acciones de reducción de oferta y las de reducción de demanda de drogas, y se llama a la conjunción de esfuerzos entre gobiernos y ONG. Esta Declaración se entregó en octubre de 2000 a todos los gobiernos de América, en la 28 sesión regular de la CICAD.

- Tres seminarios iberoamericanos, con la asistencia de todos los miembros de RIOD: el I, en Colombia, 1998; el II, en España, 1999; el III, en Bolivia, 2000. Los tres con un mismo eje: cooperación en el tema de drogas.

- Tres seminarios regionales: Centroamérica y Caribe, en Guatemala, en 1999. Cono Sur, en Argentina, en 2000. Andino y Centroamérica y Caribe, en Guatemala, en 2000. Los temas: estrategias para reducción de demanda, familia, mujer y drogas, y criterios mínimos de prevención y tratamiento.

- Dos Asambleas Generales: El Escorial (España), mayo de 1999, y Santa Cruz de la Sierra (Bolivia) noviembre de 2000.

- Documento "Criterios Mínimos para Programas de Prevención y Tratamiento", producto de uno de los seminarios regionales y complementado con las aportaciones de muchos miembros y de cuatro expertos peruanos. Un excelente derrotero científico, técnico y administrativo para las entidades que trabajan en drogodependencias y para los gobiernos nacionales y locales. La Asamblea de RIOD de 2000 lo aprobó como documento oficial de la Red, de obligatorio cumplimiento para todos los miembros.

- Presentación de RIOD en la "II Global Conference on Drug Abuse Primary Prevention", realizada en Bangkok en 1999.

Presencia e intervención en la xxviii Conferencia de la OEA. CICAD, hablando de la relación de los Estados y las Organizaciones no Estatales en materia de drogas, celebrado en Trinidad-Tobago.

\section{Estrategia de comunicación:}

- Boletín electrónico "RiodpuntoRed", que con esta edición llega a su tercer número y que se distribuye ahora a cerca de mil entidades.

- Página web: ya puede consultarse en www.riod.net y www.riod.org. Tiene una parte de acceso exclusiva para miembros de RIOD y la mayor parte de acceso general.

- Folleto y dossier de presentación: ya están elaborados, recogiendo las decisiones de la última Asamblea, se han editado y cada uno de los miem- bros podrá usarlos como apoyo a sus propias gestiones.

\section{Los apoyos}

La Delegación del Plan Nacional sobre Drogas de España convocó el I Seminario en Cartagena de Indias, acogió la idea de hacer una red iberoamericana, financió la etapa de diseño, y ha financiado y gestionado apoyos de la AECI y CICAD para todas las demás acciones. Se cuenta con su apoyo político, financiero y técnico. La RIOD forma parte de la Estrategia Nacional sobre Drogas de España 2000-2008. En todos los eventos de RIOD se ha contado con la participación de la Delegación.

La CICAD - OEA asistió a la fundación de RIOD en España y ha cofinanciado los seminarios regionales $e$ iberoamericanos. En su 28 sesión (Trinidad y Tobago, octubre 2000), la CICAD invitó a la RIOD para que se presentara ante todos los gobiernos de América.

La CICAD ha invitado a la RIOD para que participe de sus actividades, al tenor de las "Directrices para la participación de las organizaciones de la sociedad civil en las actividades de la OEA". Es un gran paso para la RIOD y un logro para el conjunto de los miembros de la red, pues uno de los objetivos centrales de RIOD es hacer interlocución con gobiernos y organismos multilaterales y la CICAD es un espacio fundamental en América en los temas de políticas y estrategias de drogas.

La AECI ha apoyado económicamente a la RIOD para la realización de los seis seminarios iberoamericanos o regionales que se han realizado. Los CIF Centros Iberoamericanos de Formación- de Cartagena de Indias, Antigua y Santa Cruz de la Sierra han sido las sedes de 4 de los seminarios de RIOD. A través del Plan Nacional sobre Drogas de España se mantiene la vinculación entre AECl y RIOD.

Se puede afirmar que desde que se creó la RIOD, no sólo ha habido estatutos, seminarios, asambleas, documentos, planes, sino que ha sido una constante realidad las múltiples interrelaciones que ha propiciado entre las entidades miembros, desde proyectos comunes, pasantías, discusiones e intercambio de informaciones en la búsqueda de propuestas conjuntas. Y de esto último hay mucho y creo que expresan una vitalidad que no es tan visible como conjunto, pero que está suponiendo un gran enriquecimiento de las entidades miembros de la RIOD. Quiero, finalmente constatar: 1) que por primera vez hay una red iberoamericana de ONGs que trabajan en drogodependencias, y 2) que en esa red están las principales ONGs de Iberoamérica en este campo.

$Y$ esto es una buena noticia y un reto para todos nosotros que lo asumimos. 
